\title{
La aspirina es eficaz en mujeres en la prevención primaria de enfermedad cerebrovascular
}

\section{Objetivo}

Evaluar la eficacia de bajas dosis de aspirina para prevenir la enfermedad cardiovascular en mujeres mayores de 45 años.

\section{Diseño}

Ensayo clínico aleatorizado y controlado.

\section{Pacientes}

Se incluyeron 39.876 mujeres profesionales de la salud sin historia de enfermedad vascular.

\section{Intervención}

Luego de un período de run-in* con placebo se asignaba a aspirina $100 \mathrm{mg}$ o placebo día por medio.

\section{Medición de resultados}

El resultado primario fue la incidencia combinada a 10 años de seguimiento de accidentes cerebrovasculares (ACV), infartos de miocardio (IM), o muertes por causa cardiovascular (CV).

\section{Resultados}

El análisis fue por intención de tratar*. No hubo diferencias significativas en el resultado primario combinado ni tampoco en la incidencia de IM o de muerte CV (ver tabla). Se observó una significativa reducción en la incidencia de ACV a expensas del tipo isquémico. La incidencia de sangrados fue superior en el grupo aspirina. En un análisis de subgrupo*, en las mayores de 65 años sí se observó una disminución del resultado combinado y de IM.

Tabla. Resultados principales

\begin{tabular}{|c|c|c|c|c|c|}
\hline Resultados de Eficacia & \begin{tabular}{|l|} 
Aspirina \\
$n=19.934$
\end{tabular} & $\begin{array}{r}\text { Placebo } \\
n=19.942\end{array}$ & AR (IC95\%) & $\mathbf{p}$ & KNT\# (IC95\%) \\
\hline Eventos Combinados & $477(2,4)$ & $522(2,6)$ & $0.91(0,80-1,03)$ & 0,13 & \\
\hline Accidente Cerebrovascular & $221(1,1)$ & $266(1,3)$ & $0,83(0,69-0,99)$ & 0,04 & $444(226-10.650)$ \\
\hline -Isquémico & $170(0,9)$ & $221(1,1)$ & $\mid 0,76(0,63-0,93)$ & 0,009 & $392(222-1.615)$ \\
\hline Hemorrágicc & o $51(0,3)$ & $41(0,2)$ & $1,24(0,82-1,87)$ & 0,31 & \\
\hline Infarto de Miocardio & $198(1,0)$ & $193(1,0)$ & $1,02(0,84-1,25)$ & 0,83 & - \\
\hline Muerte cardiovascular & $120(0,6)$ & $126(0,6)$ & $0,95(0,74-1,22)$ & 0,68 & - \\
\hline Muerte por cualquier causa & 609(3,1) & $642(3,2)$ & $0,95(0,85-1,06)$ & 0,32 & \\
\hline Resuliados de Efectos Adversos & Aspirina & Placebo & $\mathrm{ARR}$ & p & NND\#(IC95\%) \\
\hline Cualquier Sangrado & $910(4,6)$ & $751(3,8)$ & $1,22(1,10-1,34)$ & $\leq 0,001$ & $125(84-246)$ \\
\hline Sangrado que requirio transtusión & n $127(0,6)$ & $\mathbf{9 1}(0,5)$ & $1,40(1,07-1,83)$ & 0,02 & $553(305-2.787)$ \\
\hline UIlcera Péptica & $542(2,7)$ & $\mid \mathbf{4 1 3}(2,1)$ & $1,32(1,16-1,50)$ & $\leqslant 0,001$ & $154(105-287)$ \\
\hline
\end{tabular}

\section{Conclusión}

La aspirina en dosis bajas en mujeres mayores de 45 años reduce la incidencia de ACV pero no la de infartos de miocardio ni la de muerte cardiovascular.

Fuente de financiamiento: National Heart, Lung, and Blood Institute; National Cancer Institute; Bethesda; Bayer HealthCare y Natural Source Vitamin E Association.

\section{Comentario}

Las enfermedades cardiovasculares constituyen la principal causa de muerte en ambos sexos. La aspirina ha demostrado ser efectiva en el tratamiento de estas enfermedades tanto en hombres como en mujeres, sin embargo su rol en la prevención primaria es aún materia de discusión. La USPSTF ${ }^{1}$ y la American Heart Association $(\mathrm{AHA})^{2}$ recomiendan aspirina si el riesgo a diez años de tener un evento coronario es mayor al 6 y $10 \%$ respectivamente, en base a los ensayos clínicos previos. ${ }^{3.7}$ Sin embargo, la evidencia en mujeres es limitada ya que tres de estos ensayos incluyeron sólo a hombres y a que menos del $8 \%$ de los eventos en los 2 restantes ocurrieron en mujeres. Se sabe además que la aspirina tiene distinto metabolismo en hombres que en mujeres, y que la proporción de enfermedad cerebrovascular / enfermedad coronaria es superior en mujeres que en hombres. Era importante contar con este estudio impecable que aporta evidencia directa del verdadero impacto de la aspirina en mujeres.

Es posible que el estudio no detectara diferencias en el resultado primario combinado porque fue diseñado para encontrar una ambiciosa diferencia del $25 \%$ para esta población de muy bajo riesgo de eventos CV a 10 años. Sin embargo, sí encontró beneficios de la aspirina en un importante resultado secundario como ACV (NNT* de 444); pero no se encontraron diferencias en IM ni en muerte CV. Este beneficio debería ser sopesado con el aumento de la incidencia de cualquier tipo de sangrado, desde leves a graves (el NND de sangrado que requiriera transfusión fue 553). Podríamos decir que para prevenir $1 \mathrm{ACV}$ habría que tratar a 444 mujeres y transfundir a 3 ó 4 por sangrados durante diez años.
El análisis del subgrupo* de mayores de 65 años mostró una reducción significativa no sólo de ACV sino también de IM y de eventos combinados, y un aumento de sangrados graves. En esta población de mayor riesgo cabría esperar un mayor beneficio pero no hay que olvidar que los análisis de subgrupos no están diseñados para contestar el objetivo primario y no cambian la práctica sino plantean hipótesis para futuros ensayos. Los resultados de este estudio parecen contrastar con los obtenidos en ensayos de prevención primaria en hombres en los cuales se halló una significativa reducción de IM pero no de $\mathrm{ACV}^{3-7}$. Sin embargo para distinguir la eficacia entre hombres y mujeres deberíamos comparar riesgos de eventos similares y también dosis similares. En el presente estudio, se usó un diferente esquema de aspirina $(100 \mathrm{mg}$ en días alternos), dosis inferior a la de cualquier ensayo previo de aspirina. Esto constituye quizás la mayor limitación de este estudio y dificulta cualquier comparación.

\section{Conclusión del comentador}

Las implicancias de este estudio para nuestra práctica no parecen ser demasiadas dado que la población estudiada fue de bajo riesgo y no tiene actualmente indicación de recibir aspirina. La modesta eficacia en la reducción de ACV es a costa de no pocos sangrados. Por otro lado, no descarta que la aspirina pueda llegar a ser útil en mujeres de más del $5-9 \%$ de riesgo de eventos CV a 10 años, grupo para el que aun no contamos con evidencias directas.

\section{Dr. Sergio Dominelli [ Unidad de Medicina Familiar y Preventiva. Hospital Italiano de Buenos Aires. ]}

* ver glosario

Dominelli S. La aspirina es eficaz en mujeres en la prevención primaria de enfermedad cerebrovascular. Evid actual pract ambul 2005;8:69. Ridker PM, Cook $\mathrm{NR}$, Lee IM, et al. A randomized trial of low-dose aspirin in the primary prevention of cardiovascular disease in women. $\mathrm{N}$ Engl $\mathrm{J}$ Med.2005; 352 (13):1293-304. PMID: 15753114

\section{Referencias}

1. U.S. Preventive Services Task Force. Aspirin for the primary prevention of cardiovascular events: recommendations and rationale. Ann Int Med 2002;136(2):157-60.

2. Hennekens CH, Dyken ML, Fuster V. Aspirin as a therapeutic agent in cardiovascular disease: a statement for healthcare professionals from the American Heart Association. Circulation 1997;96:2751-3.

3. Steering Committee of the Physicians' Health Study Research Group. Final report on the aspirin component of the ongoing Physicians' Health Study. N Engl J Med 1989;321:129-135. 4. Peto R, Gray R, Collins R, et al. Randomised trial of prophylactic daily aspirin in British male doctors. Br Med J (Clin Res Ed) 1988;296:313-316.

5. The Medical Research Council's General Practice Research Framework. Thrombosis prevention trial: randomised trial of low-intensity oral anticoagulation with warfarin and low-dose aspirin in the primary prevention of ischaemic heart disease in men at increased risk. Lancet 1998;351:233-241.

6. Hansson L, Zanchetti A, Carruthers SG, et al. Effects of intensive blood-pressure lowering and low-dose aspirin in patients with hypertension: principal results of the Hypertension Optimal Treatment (HOT) randomised trial. Lancet 1998;351:1755-1762.

7. Collaborative Group of the Primary Prevention Project. Low-dose aspirin and vitamin E in people at cardiovascular risk: a randomised trial in general practice. Lancet 2001;357:89-95. 